\title{
Design of DTCWT-DWT Image Compressor-Decompressor with Companding Algorithm
}

\author{
${ }^{1}$ Venkateshappa, ${ }^{2}$ Cyril Prasanna Raj P \\ ${ }^{1}$ Research Scholar, Department of Electronics \&Communication Engineering, M S Engineering \\ College, Bengaluru, 562110, Karnataka, India.; \\ 2. Professor, Dean R\&D, Department of Electronics \&Communication Engineering, M S Engineering \\ College, Bengaluru, 562110, Karnataka, India; \\ Venkat_harishith@rediffmail.com; cyril@msec.ac.in;
}

\begin{abstract}
Discrete Wavelet Transform (DWT) has demonstrated advantages in image compression due to its time-frequency resolution property. Dual Tree Complex Wavelet Transform (DTCWT) in addition to the advantages of DWT supports additional properties such as directionality and shift invariant. DTCWT has coefficient redundancy of $4: 1$ or $2^{\mathrm{m}}: 1$ in $\mathrm{m}$ dimensions thus is four time complex than DWT computation. In this work, DTCWT based image compression algorithm is proposed that performs decomposition using DTCWT and reconstruction is carried out using DWT. The DTCWT bands are companded and encoded using entropy encoder and the quantization of the sub bands introduces minimum loss. The proposed algorithm achieves $77 \%$ compression with $40 \mathrm{~dB}$ PSNR thus suitable for real time image compression. DTCWT filters are appropriately selected to achieve perfect reconstruction.
\end{abstract}

Keywords: Image compression, DTCWT, SPIHT, DWT, companding.

\section{Introduction}

Image compression plays an important role in today's multimedia applications by compressing raw image data with compression ratios greater than 100. Image compression standards such as JPEG 2000 [1] and video compression standards such as H.265 [2] [3] recommend use of wavelets for image transformation into frequency sub bands. Encoding techniques [4] such as arithmetic coding, SPIHT encoding and variable length coding encodes wavelet bands into binary bits. The process of quantization and thresholding of wavelet bands prior to encoding determines the compression ratios [5]. The sub bands of Discrete Wavelet Transform (DWT) such as LL, LH, HL and HH (with level 1) consists of intensity, vertical, horizontal and diagonal features (edges) respectively. Given an input image of size $\mathrm{N} \times \mathrm{N}$ after level $12 \mathrm{D}$ DWT decomposition has four sub bands each of size N/2 $\times \mathrm{N} / 2$. Considering the LL band (dominant information in terms of intensity) and significant features from all three sub bands, SPIHT encoding logic encodes the significant information achieving compression. The significant features from higher sub bands contain edge information in $0^{\circ}, 90^{\circ}$ and $45^{\circ}$ directions. The input image to be compressed may contain edge information in all other orientations apart from $0^{\circ}$, $90^{\circ}$ and $45^{\circ}$. In order to capture the significant orientations such as $0^{\circ}, \pm 15^{\circ}, \pm 45^{\circ}$ and $\pm 75^{\circ}$ Dual Tree Complex Wavelet Transforms (DTCWT) has been proposed by Kingsbury [6]. Discussion on DTCWT demonstrating its advantages in image processing applications with regard to shift insensitive and 
Venkateshappa, Cyril Prasanna Raj P; Design of DTCWT-DWT Image Compressor-Decompressor with Companding Algorithm. Advances in Image and Video Processing, Volume 5 No 1, February (2017); pp: 1-12 directionality are presented in [2-6]. Li Hue Fang et al. [7] have proposed a new image compression algorithm that is based on DT-CWT and SPIHT. To encode DTCWT bands modified SPIHT algorithm is presented in their work. DTCWT and modified SPIHT based compression increases computation complexity. Fowler et al. [8] propose embedded wavelet-based coder with noise-shaping technique to eliminate redundancy in DTCWT sub bands thus resulting compression of $0.1 \mathrm{bpp}$ and maximum PSNR of $40 \mathrm{~dB}$. Chuo-Ling Chang et al.[9] propose a direction-adaptive DWT (DA-DWT) that locally adapts the filtering directions to image content based on directional lifting. With the adaptive transform, energy compaction is improved for sharp image features. The analysis indicates that the proposed DA-DWT is more effective than other lifting-based approaches. Jingyu Yang et al.[10] propose an image coding scheme using 2-D anisotropic dual-tree discrete wavelet transform (DDWT) with coefficients rearranged to improve zero-tree relationship for efficient SPIHT encoding. The algorithm achieves PSNR of $32.54 \mathrm{~dB}$ at $0.1 \mathrm{bpp}$. Satellite image enhancement improving PSNR is carried out using DTCWT by interpolating the higher bands of DTCWT and the input image to achieve high resolution images [11]. Medical image denoising is carried out by shrinking DTCWT bands using Wiener filter that has demonstrated improvement in denoising results by balancing between accuracy and smoothness [12]. DTCWT bands have been effectively utilized to perform image processing and image compression. DTCWT computation based on Kingsbury proposes real and complex filters to compute orientations in six orientations. 2D DTCWT level-1 decomposition requires four filters for row processing and sixteen filters for column processing [13]. Each decomposition level consists of low pass bands (real and imaginary) and six high frequency sub bands (real and imaginary). DTCWT sub bands capture significant edge information in more than three directions and hence have advantages compared with DWT. DTCWT employs DWT filters for computation of real and imaginary sub bands. With the number filters in DTCWT more than the number of filters in DWT, computation complexity of DTCWT both at the source and destination is intensive. Use of DTCWT sub bands for information extraction during decomposition and encoding the information using entropy coding techniques achieves compression. At the destination, the received bits can be decompressed using DWT filters instead of using DTCWT filters, thus reducing the complexity of decoder. In this work, the compressor is designed using DTCWT and entropy coding; the reconstruction is carried out using DWT in place of DTCWT.

Section II discusses image compression algorithm using DTCWT, section III discusses proposed algorithm, section IV presents discussion on compression results and section $\mathrm{V}$ is conclusion.

\section{Image Compression Algorithm}

The 2D DTCWT architecture based on the discussions in [14] is shown in Figure 1. The input image is processed along the rows with the filter pair $F_{h}=\left\{H_{0 a}, H_{0 b}\right\}$ and $F_{g}=\left\{H_{1 a}, H_{1 b}\right\}$, the filters satisfy the conditions $\mathrm{H} 1 \mathrm{x}=\mathrm{HOx}(\mathrm{n}-1)$. The four outputs of row processing are further processed along the columns with the set of two filter banks to obtain sixteen bands. 


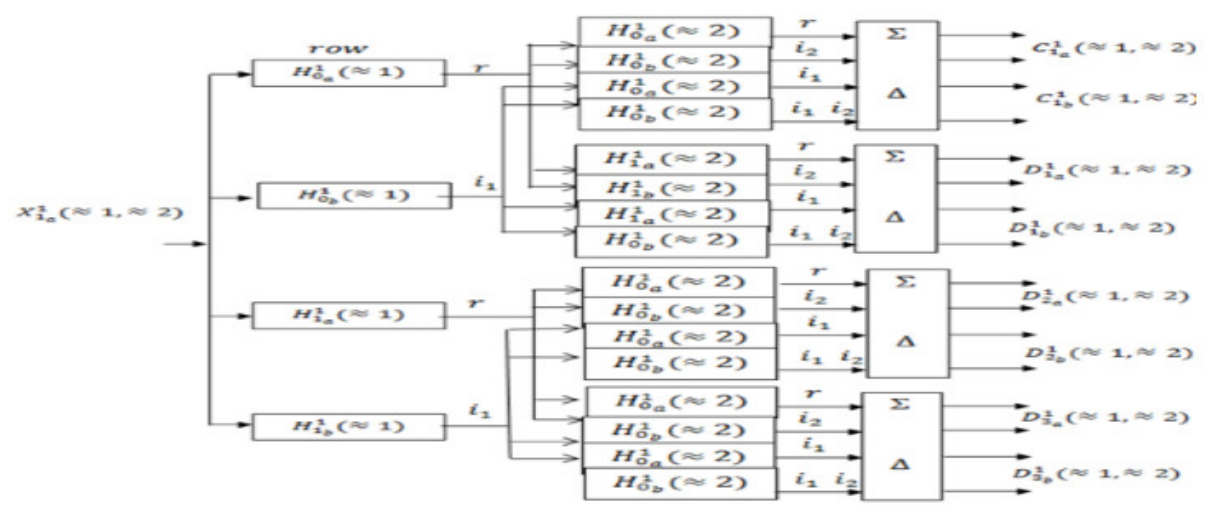

Figure 1: Single Stage Dual Trees CWT

Decomposition of input image using DTCWT consists of real and imaginary sub bands [15] denoted as ( $\mathbf{w} 1+\mathbf{j w 2}$.), the real part of decomposition $\mathbf{w} 1$ is given as in Eq. (4),

$$
W_{1}=\frac{1}{2} \quad\left[\begin{array}{rr}
1 & -1 \\
1 & 1
\end{array}\right]\left[\begin{array}{l}
F_{h h} \\
F_{g g}
\end{array}\right] X
$$

The imaginary part of image decomposition $\mathbf{w} 2$ is given as in Eq. (5),

$$
W_{2}=\frac{1}{2}\left[\begin{array}{cc}
1 & 1 \\
1 & -1
\end{array}\right]\left[\begin{array}{l}
F_{g h} \\
F_{h g}
\end{array}\right] X
$$

Fhh denotes the filters pairs $\left\{\mathrm{H}_{0 a}, \mathrm{H}_{0 b}\right\}, \mathrm{H}_{0 \mathrm{a}}$ filter is used along the rows and $\mathrm{H}_{0 \mathrm{~b}}$ is used along the column. Similarly the other filter pairs Fgg $\left\{\mathrm{H}_{1 \mathrm{a}}, \mathrm{H}_{1 b}\right\}$, Fhg $\left\{\mathrm{H}_{0 \mathrm{a}}, \mathrm{H}_{1 b}\right\}$ and Fgh $\left\{\mathrm{H}_{1 b}, \mathrm{H}_{0 \mathrm{a}}\right\}$ are used along rows and columns.

\subsection{Transformation Algorithm}

In DWT decomposition, the input image after level-1 decomposition produces four bands, one low pass (LL) and three high pass ( $\mathrm{LH}, \mathrm{HL}$ and $\mathrm{HH}$ ). The level-1 high frequency bands are represented by $\boldsymbol{\Psi}_{\mathbf{1}}(\boldsymbol{X}, \boldsymbol{Y})=\boldsymbol{\varphi}(\boldsymbol{X}) \boldsymbol{\psi}(\boldsymbol{Y}) \quad$ (LH wavelet), $\boldsymbol{\Psi}_{\mathbf{2}}(\boldsymbol{X}, \boldsymbol{Y})=\boldsymbol{\psi}(\boldsymbol{X}) \boldsymbol{\varphi}(\boldsymbol{Y}) \quad$ (HL wavelet) and $\boldsymbol{\Psi}_{\mathbf{3}}(\boldsymbol{X}, \boldsymbol{Y})=$ $\boldsymbol{\psi}(\boldsymbol{X}) \boldsymbol{\psi}(\boldsymbol{Y})$ (HH wavelet), $\omega($.$) is low pass function and \boldsymbol{\psi}($.$) is the high pass function, \mathrm{x}, \mathrm{y}$ indicate the dimensions. The dual tree complex wavelets sub bands are denoted by $\boldsymbol{\psi}(\boldsymbol{X}, \boldsymbol{Y})=\boldsymbol{\psi}(\boldsymbol{X}) \boldsymbol{\psi}(\boldsymbol{Y})$ where $\psi($.$) is complex wavelet given by \psi()=.\psi_{\mathrm{h}}()+.j \psi_{\mathrm{g}}($.$) , Substituting the complex wavelet, the$ DTCWT output is expressed as in Eq. (1),

$$
\begin{aligned}
& \psi(X, Y)=\left[\Psi_{h}(X)+j \Psi_{g}(X)\right]\left[\Psi_{h}(Y)+j \Psi_{g}(Y)\right] \\
= & \psi_{h}(X) \Psi_{h}(Y)-\Psi_{g}(X) \Psi_{g}(Y)+j\left[\Psi_{g}(X) \Psi_{h}(Y)+\psi_{h}(X) \Psi_{g}(Y)\right]
\end{aligned}
$$

A set of six complex wavelets of DTCWT as defined by Kingsbury is given in Eq. (2),

$$
\begin{array}{r}
\Psi_{\mathrm{i}}(X, Y)=1 / \sqrt{2}\left(\Psi_{1}, i(X, Y)-\Psi_{2}, i(X, Y)\right) \\
\Psi_{\mathrm{i}+3}(X, Y)=1 / \sqrt{2}\left(\Psi_{1}, i(X, Y)+\Psi_{2}, i(X, Y)\right) \\
\Psi_{\mathrm{i}}(X, Y)=1 / \sqrt{2}\left(\Psi_{3}, i(X, Y)+\Psi_{4}, i(X, Y)\right) \\
\Psi_{\mathrm{i}+3}(X, Y)=1 / \sqrt{2}\left(\Psi_{3}, i(X, Y)+\Psi_{4}, i(X, Y)\right)
\end{array}
$$

Eq. 2(a) and 2(b) represents the real and Eq. 2(c) and 2(d) represents the complex wavelets. Considering Eq. 2, for $\mathrm{i}=1$, 2, 3 the 2-D wavelet bases (real) and 2-D wavelet bases (imaginary) are presented in Table 1. 
Table 1

\begin{tabular}{|l|l|l|l|}
\hline \multirow{2}{*}{ i=1 } & Real & $\Psi_{1,1}(X, Y)=\varphi_{h}(X) \psi_{h}(Y)$ & $\Psi_{2,1}(X, Y)=\varphi_{g}(X) \psi_{g}(Y)$ \\
\cline { 2 - 4 } & Imaginary & $\Psi_{3,1}(X, Y)=\varphi_{g}(X) \psi_{h}(Y)$ & $\Psi_{4,1}(X, Y)=\varphi_{h}(X) \psi_{g}(Y)$ \\
\hline \multirow{2}{*}{ i=2 } & Real & $\Psi_{1,2}(X, Y)=\psi_{h}(X) \varphi_{h}(Y)$ & $\Psi_{2,2}(X, Y)=\psi_{g}(X) \varphi_{g}(Y)$ \\
\cline { 2 - 4 } & Imaginary & $\Psi_{3,2}(X, Y)=\psi_{g}(X) \varphi_{h}(Y)$ & $\Psi_{4,2}(X, Y)=\psi_{h}(X) \varphi_{g}(Y)$ \\
\hline I=3 & Real & $\Psi_{1,3}(X, Y)=\psi_{h}(X) \psi_{h}(Y)$ & $\Psi_{2,3}(X, Y)=\psi_{g}(X) \psi_{g}(Y)$ \\
\cline { 2 - 4 } & Imaginary & $\Psi_{3,3}(X, Y)=\psi_{g}(X) \psi_{h}(Y)$ & $\Psi_{4,3}(X, Y)=\psi_{h}(X) \psi_{g}(Y)$ \\
\hline
\end{tabular}

The set of six wavelets defined above are significant in isolating six orientations in the input image along specific direction and are contained in the twelve sub bands without any artifacts. Considering the term $\boldsymbol{\psi}_{\boldsymbol{h}}(\boldsymbol{X}) \boldsymbol{\psi}_{\boldsymbol{h}}(\boldsymbol{Y})$ which is computed using the filter pair $\left\{\mathrm{H}_{0 \mathrm{a}}, \mathrm{H}_{0 \mathrm{~b}}\right\}$, which is equivalent to the term $\boldsymbol{\Psi}_{3}(\boldsymbol{X}, \boldsymbol{Y})=\boldsymbol{\psi}(\boldsymbol{X}) \boldsymbol{\psi}(\boldsymbol{Y}) \quad$ (or $\mathrm{HH}$ wavelet band) computed using DWT. The term $\Psi_{\mathrm{g}}(\mathrm{x}) \psi_{\mathrm{g}}(\mathrm{y})$ computed using filter pair $\left\{\mathrm{H}_{1 \mathrm{a}}, \mathrm{H}_{1 b}\right\}$ is the imaginary term of $\boldsymbol{\psi}_{\boldsymbol{h}}(\boldsymbol{X}) \boldsymbol{\psi}_{\boldsymbol{h}}(\boldsymbol{Y})$. It is also known that the filter $\mathrm{H} 1 \mathrm{x}=\mathrm{HOx}(\mathrm{n}-1)$, there will be redundancy in the twelve sub bands, thus reconstruction of original image with inverse DTCWT requires either the real or the imaginary band alone. Performing inverse DTCWT with either the real or imaginary bands will not contain all the required analytic information in the reconstructed image. Eliminating redundant bands of DTCWT and performing reconstruction using DTCWT will not lead to perfect reconstruction. In this proposed work, instead of discarding the real or imaginary sub bands, the information from the entire twelve sub bands are captured and confined to three bands and are termed as $\mathrm{LH}_{\mathrm{D}}, \mathrm{HL}_{\mathrm{D}}$ and $\mathrm{HH}_{\mathrm{D}}$ representing optimum bands derived from DTCWT sub bands. For reconstruction of original image from DWT is recommended instead of DTCWT. The reconstruction filters used in DWT are $\left\{\mathrm{H}^{\prime}{ }_{0 a}, \mathrm{H}^{\prime}{ }_{0 b}\right\}$ which are the synthesis filters of DTCWT. In summary, in the proposed design, forward DTCWT transform is carried out with Eq. (3), and inverse DWT transform is carried out with Eq. (4) [16].

$$
\begin{gathered}
F_{02 D}=\frac{1}{\sqrt{8}}\left[\begin{array}{rrrc}
1 & -1 & \\
1 & 1 & & \\
& & 1 & 1 \\
1 & -1
\end{array}\right]\left[\begin{array}{l}
F_{h h} \\
F_{g g} \\
F_{g h} \\
F_{h g}
\end{array}\right] \\
F_{2 D}^{-1}=\frac{1}{2}\left[\begin{array}{lll}
F_{h h}^{-1} & F_{g g}^{-1}
\end{array}\right]\left[\begin{array}{rr}
1 & 1 \\
-1 & 1
\end{array}\right]
\end{gathered}
$$

\subsection{Encoding Algorithm}

Multilevel decomposition with DTCWT leads to a pyramidal structure as shown in Figure 2. The input image of $\mathrm{N} \times \mathrm{N}$ after level 1 DTCWT produces six real sub bands and two low pass bands each of size $\mathrm{N} / 2 \times \mathrm{N} / 2$. Level-2 decomposition decomposes low pass bands to eight real sub bands each of size N/4 $x \mathrm{~N} / 4$. Thus after three levels of DTCWT decomposition, there will be eight real sub bands of size N/8 $x \mathrm{~N} / 8$ at level-3, six real sub bands of size N/4 $\times \mathrm{N} / 4$ at level-2 and six real sub bands of size N/2 $\times \mathrm{N} / 2$. The low pass bands at level- 3 capture the DC components of original image at N/8 $\times$ N/8 resolution and all other sub bands capture the orientations of edge information in the input image. Image compression is achieved by encoding the DC components from the low pass bands and with significant edges from the high frequency sub bands from three levels. One of the methods for encoding is to use Set partitioning in hierarchical trees (SPIHT). 


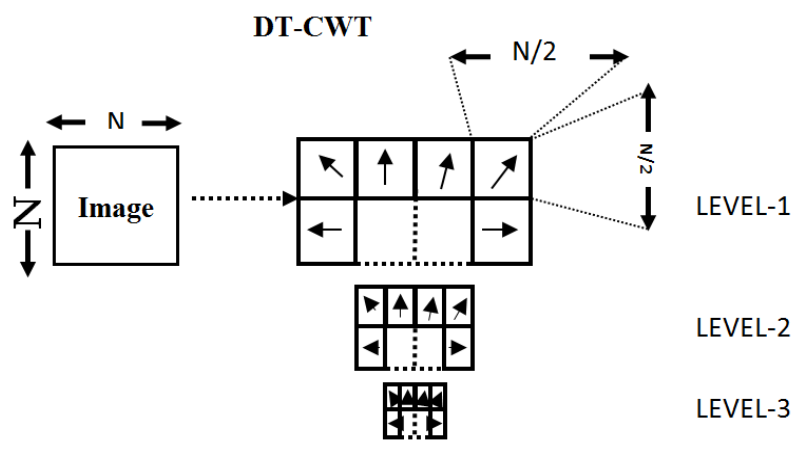

Figure 2: Three level DTCWT decomposition

SPIHT is an image encoding algorithm identifies similarities across wavelet sub bands encodes most significant transform coefficients first for transmission and during refinement process other wavelet coefficients are progressively encoded [17][18][19].

The algorithm encodes the wavelet bands into stream of bits in two stages: sorting and refinement stage. List of Significant Pixels (LSP) an empty list initially; List of Insignificant Pixels (LIP) that stores all wavelet coefficients and List of Insignificant Sets (LIS) that stores the tree roots at the lowest band are three lists in SPIHT. During the sorting pass, the wavelet coefficients in the LIP are selected and moved to LSP if they are significant and above a set threshold. LIS is updated based on partitioning sorting algorithm. In the refinement pass, the coefficients in LSP are searched and encoded to ' 1 ' or ' 0 ' based on a set threshold. The threshold level is scaled by 2 every pass (sorting and refinement) and the algorithm iteratively encode until the threshold level reaches a desired level. Flow chart shown in Figure 3 illustrates the data flow diagram in SPIHT encoder.

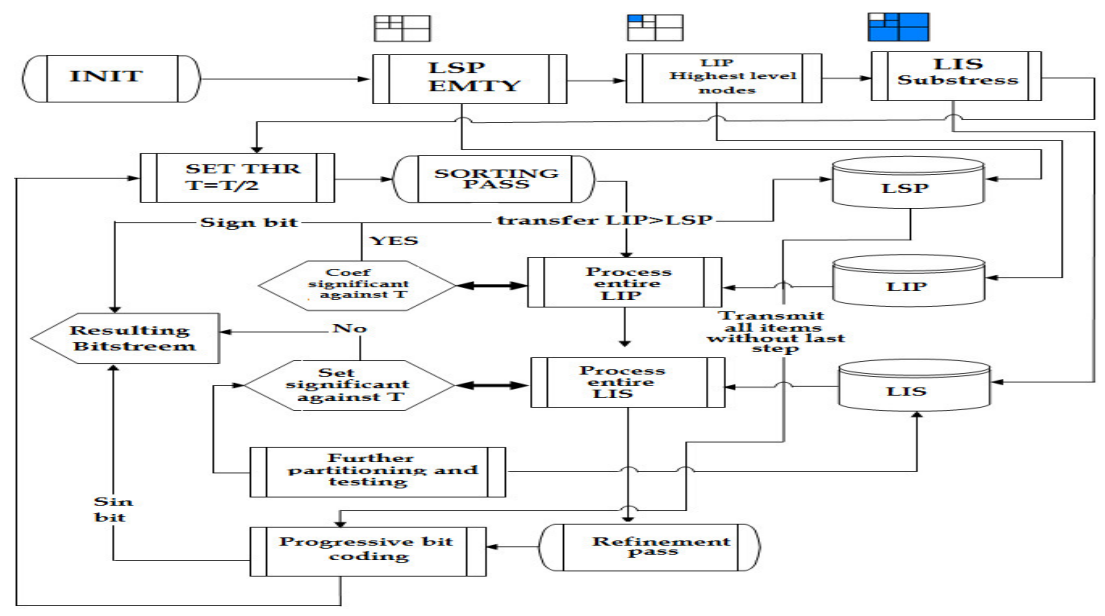

Figure 3: SPIHT algorithm flow graph

In DWT based image compression, at every level there would be three bands and SPIHT algorithm encodes the low pass and high pass images considering the significant pixels from each band at multiple levels. In the proposed work, the DTCWT bands that contain the edge orientations in six directions contain more information than compared with DWT bands. Thus the orientations in all these six bands are companded into three bands at each level. The companded bands are encoded using SPIHT algorithm. A detailed discussion on reducing DTCWT bands to equivalents of DWT bands is resented in next section. SPIHT encoding is based on significant features and threshold levels. In this work, the threshold levels are determined by considering the energy gradients in the DTCWT bands in all six directions (shown in Figure 4). The maximum gradient and the energy level of the gradients are considered in setting the threshold levels for SPIHT algorithm. 

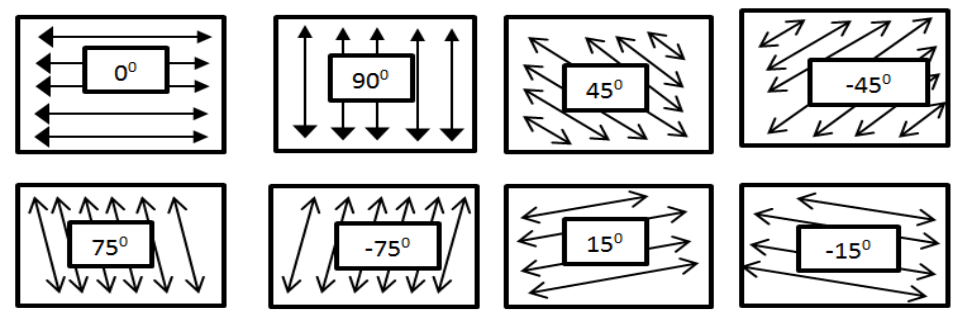

Figure 4: Orientations in DTCWT sub bands

The gradients of an image [20] are computed as in Eq. (5),

$$
\frac{\partial \mathrm{f}(\mathrm{x}, \mathrm{y})}{\partial \mathrm{x}}=\nabla_{\mathrm{x}}=\frac{\mathrm{f}\left(\mathrm{x}+\mathrm{d}_{\mathrm{x}, \mathrm{y}}\right)-\mathrm{f}(\mathrm{x}, \mathrm{y})}{\mathrm{d}_{\mathrm{x}}}, \quad \frac{\partial \mathrm{f}(\mathrm{x}, \mathrm{y})}{\partial \mathrm{y}}=\nabla_{\mathrm{y}}=\frac{\mathrm{f}\left(\mathrm{x}, \mathrm{y}+\mathrm{d}_{\mathrm{y}}\right)-\mathrm{f}(\mathrm{x}, \mathrm{y})}{\mathrm{d}_{\mathrm{x}}}
$$

Where $\mathrm{dx}$ and $\mathrm{dy}$ measure distance $\mathrm{x}$ and $\mathrm{y}$ directions respectively. From the gradients the maximum gradient is identified from each of the six bands at all three levels, the gradient that is minimum is set as the threshold. Thus during SPIHT encoding phase the significant pixel information encodes even the least significant DTCWT coefficient. The significance of each edge orientation is determined by computing the intensity along the directions. If the sum of intensity is significant, the edge point is retained. From all the eight sub bands three sub bands are determined.

\section{Proposed Algorithm}

Figure 5 shows the proposed image compression algorithm. In the proposed algorithm, the input image is decomposed into sub bands using DTCWT. Three-level decomposition produces twenty sub bands, from each level six sub bands three bands are computed with the companding algorithm. The companding algorithm reduces all the DTCWT bands to ten DWT equivalent bands.

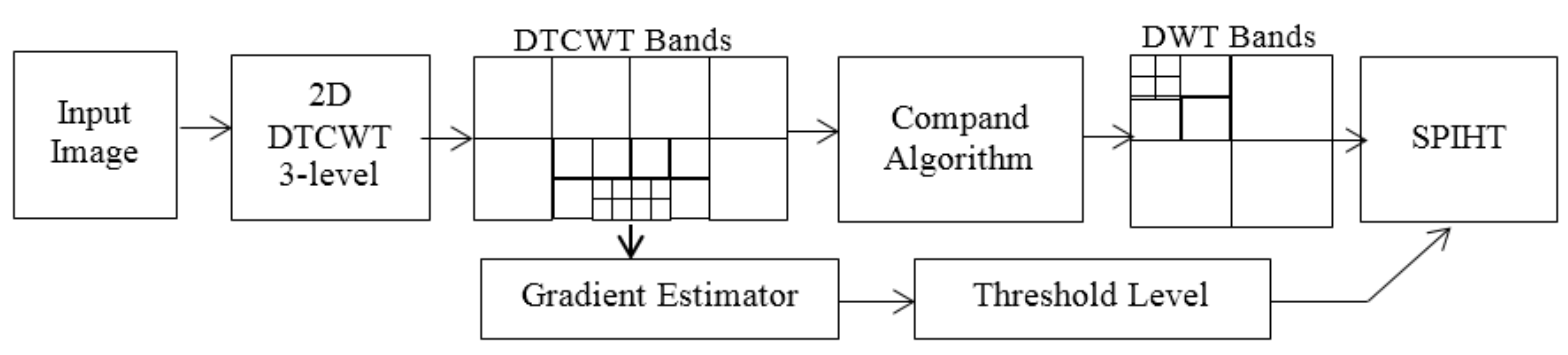

Figure 5 Proposed image compression block diagram

From the DTCWT bands gradient operation estimates intensity along all orientations and Threshold levels are set as discussed previously and SPIHT algorithm compresses the DTCWT equivalent DWT bands with the set threshold levels to compressed bit stream.

\subsection{Companding algorithm}

The input image is transformed using four filters that operate in parallel on the input data, each filter consists of wavelet filters of length-10. Table 2 shows the filter coefficients for the first stage decomposition and Table 2 shows the filter coefficients for second and third stage decomposition. The reconstruction is carried out using inverse DWT, the reconstruction filters are obtained by reversing the alternate coefficients of decomposition filters. The filters in tree a are reversed for inverse DWT filters. 
Table 2 DTCWT filters for level-1

\begin{tabular}{|c|c|c|c|}
\hline \multicolumn{2}{|c|}{ Tree $\mathrm{a}$} & \multicolumn{2}{c|}{ Tree b } \\
\hline $\mathrm{H}_{0 \mathrm{a}}$ & $\mathrm{H}_{1 \mathrm{a}}$ & $\mathrm{H}_{0 \mathrm{~b}}$ & $\mathrm{H}_{1 \mathrm{~b}}$ \\
\hline 0 & 0 & 0.01122679 & 0 \\
\hline-0.08838834 & -0.01122679 & 0.01122679 & 0 \\
\hline 0.08838834 & 0.01122679 & -0.08838834 & -0.08838834 \\
\hline 0.69587998 & 0.08838834 & 0.08838834 & -0.08838834 \\
\hline 0.69587998 & 0.08838834 & 0.69587998 & 0.69587998 \\
\hline 0.08838834 & -0.69587998 & 0.69587998 & -0.69587998 \\
\hline-0.08838834 & 0.69587998 & 0.08838834 & 0.08838834 \\
\hline 0.01122679 & -0.08838834 & -0.08838834 & 0.08838834 \\
\hline 0.01122679 & -0.08838834 & 0 & 0.01122679 \\
\hline 0 & 0 & 0 & -0.01122679 \\
\hline
\end{tabular}

Table 3 DTCWT filters for higher levels

\begin{tabular}{|c|c|c|c|}
\hline \multicolumn{2}{|c|}{ Tree $\mathrm{a}$} & \multicolumn{2}{c|}{ Tree $\mathrm{b}$} \\
\hline $\mathrm{H}_{\text {0oa }}$ & $\mathrm{H}_{\text {o1a }}$ & $\mathrm{H}_{00 \mathrm{~b}}$ & $\mathrm{H}_{01 \mathrm{~b}}$ \\
\hline 0 & 0 & 0 & 0.03516384 \\
\hline 0.03516384 & 0 & 0 & 0 \\
\hline-0.08832942 & & & 0.08832942 \\
\hline 0.023389032 & 0 & 0 & 0.023389032 \\
\hline 0.76027237 & 0.58751830 & 0.58751830 & -0.76027237 \\
\hline 0.58751830 & -0.76027237 & 0.76027237 & 0.58751830 \\
\hline 0 & 0.023389032 & 0.023389032 & 0 \\
\hline-0.11430184 & 0.08832942 & -0.08832942 & -0.11430184 \\
\hline 0 & 0 & 0 & 0 \\
\hline 0 & -0.03516384 & 0.03516384 & 0 \\
\hline
\end{tabular}

The DTCWT requires four filters $\left\{\mathrm{H}_{0 \mathrm{a}}, \mathrm{H}_{0 b}\right\}$ and $\left\{\mathrm{H}_{1 \mathrm{a}}, \mathrm{H}_{1 b}\right\}$ in the first stage for row processing satisfies the property as in Eq. (6),

$$
\begin{array}{ccc}
H_{0 a}^{1}(Z)= & H_{0}(Z), & H_{0 a}^{1}(Z)=H_{1}(Z)=Z^{-1} H_{0}\left(-Z^{-1}\right) \\
H_{0 b}^{1}(Z)=Z^{-1} & H_{0}(Z), & H_{0 b}^{1}(Z)=Z^{-1} \quad H_{1}(Z)=Z^{-2} H_{0}\left(-Z^{-1}\right)
\end{array}
$$

Let $\boldsymbol{A}_{\mathbf{1}}(\boldsymbol{Z})=\boldsymbol{H}_{\mathbf{0}}^{\mathbf{1}}(\boldsymbol{Z}), \quad \boldsymbol{B}_{\mathbf{1}}(\boldsymbol{Z})=\boldsymbol{H}_{\mathbf{1}}^{\mathbf{1}}(\boldsymbol{Z}), \quad$ and the cascaded filter bank for $\mathrm{N}$ levels $(\mathrm{j}=1,2,3, \ldots . \mathrm{N})$ can be represented as in Eq. (7),

$$
\begin{aligned}
A^{j}(Z) & =H_{0}^{1}(Z) \cdots H_{0}^{j-1}\left(Z^{2 j-2}\right) H_{0}^{j}\left(Z^{2 j-1}\right) \\
B^{j}(Z) & =H_{0}^{1}(Z) \cdots H_{0}^{j-1}\left(Z^{2 j-2}\right) H_{1}^{j}\left(Z^{2 j-1}\right)
\end{aligned}
$$

Denoting the DTCWT output of approximation and detail coefficients as $\{C, D\}$ for a given input $S$, at level $\mathrm{j}$, the four outputs are represented as in Eq. (8), 


$$
\begin{aligned}
& C_{a / b}^{j}\left(Z_{1}, Z_{2}\right)=\left(2^{J} \downarrow\right)\left(\left(A_{a}^{j}\left(Z_{1}\right) \pm j\left(A_{b}^{j}\left(Z_{1}\right)\right)\left(A_{a}^{j}\left(Z_{2}\right)+j\left(A_{b}^{j}\left(Z_{2}\right)\right) S\left(Z_{1}, Z_{2}\right)\right)\right.\right. \\
& D_{1 a / b}^{j}\left(Z_{1}, Z_{2}\right)=\left(2^{J} \downarrow\right)\left(\left(A_{a}^{j}\left(Z_{1}\right) \pm j\left(A_{b}^{j}\left(Z_{1}\right)\right)\left(B_{a}^{j}\left(Z_{2}\right)+j\left(B_{b}^{j}\left(Z_{2}\right)\right) S\left(Z_{1}, Z_{2}\right)\right)\right.\right. \\
& D_{2 a / b}^{j}\left(Z_{1}, Z_{2}\right)=\left(2^{J} \downarrow\right)\left(\left(B_{a}^{j}\left(Z_{1}\right) \pm j\left(B_{b}^{j}\left(Z_{1}\right)\right)\left(A_{a}^{j}\left(Z_{2}\right)+j\left(A_{b}^{j}\left(Z_{2}\right)\right) S\left(Z_{1}, Z_{2}\right)\right)\right.\right. \\
& D_{3 a b}^{j}\left(Z_{1}, Z_{2}\right)=\left(2^{J} \downarrow\right)\left(\left(B_{a}^{j}\left(Z_{1}\right) \pm j\left(B_{b}^{j}\left(Z_{1}\right)\right)\left(B_{a}^{j}\left(Z_{2}\right)+j\left(B_{b}^{j}\left(Z_{2}\right)\right) S\left(Z_{1}, Z_{2}\right)\right)\right.\right.
\end{aligned}
$$

The subscript terms $\mathrm{a} / \mathrm{b}$ is associated with the sign $( \pm)$ in the first term, $\mathrm{C}_{\mathrm{a}}$ and $\mathrm{C}_{\mathrm{b}} \mathrm{b}_{\mathrm{b}}$ represents the two complex low-pass sub bands, $\mathrm{D}_{1 \mathrm{a}}\left(-15^{\circ}\right), \mathrm{D}_{1 \mathrm{~b}}\left(+15^{\circ}\right), \mathrm{D}_{2 \mathrm{a}}\left(-75^{\circ}\right), \mathrm{D}_{2 \mathrm{~b}}\left(+75^{\circ}\right), \mathrm{D}_{3 \mathrm{a}}\left(-45^{\circ}\right)$ and $\mathrm{D}_{3 \mathrm{~b}}\left(+45^{\circ}\right)$ represents the six complex high pass sub bands. The two complex low pass bands are companded to a single LL band as shown in Figure 6 . The real or imaginary part of $\mathrm{C}^{\mathrm{j}}$ is selected and further the average of the two selected bands gives rise to LL band as this band comprises of maximum information from both the LL bands and thus redundancy is reduced

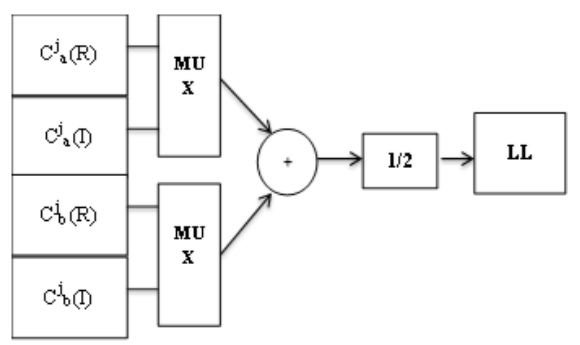

Figure 6: Companding algorithms for LL band

Similarly from the six complex sub bands, the companding algorithm captures the information in all these bands and compands to three bands termed as $\mathrm{LH}, \mathrm{HL}$ and $\mathrm{HH}$ band. Figure 7 shows the companding algorithm for generation of LH band. The real or imaginary band is selected, the gradient operation computes the gradients in $\left( \pm 15^{\circ}\right)$ using Eq. (5). The maximum gradient intensity is determined and is scaled by a factor of 2 to set the threshold level. With the set threshold the selected band is processed to retain the significant features. Two images are obtained that contain features in $\left(-15^{\circ}\right) \&\left(+15^{\circ}\right)$. These two images are fused by computing the average intensities to obtain the $\mathrm{LH}$ band. The LH band generated consists of orientations in both $\left( \pm 15^{\circ}\right)$ that were captured from DTCWT bands.

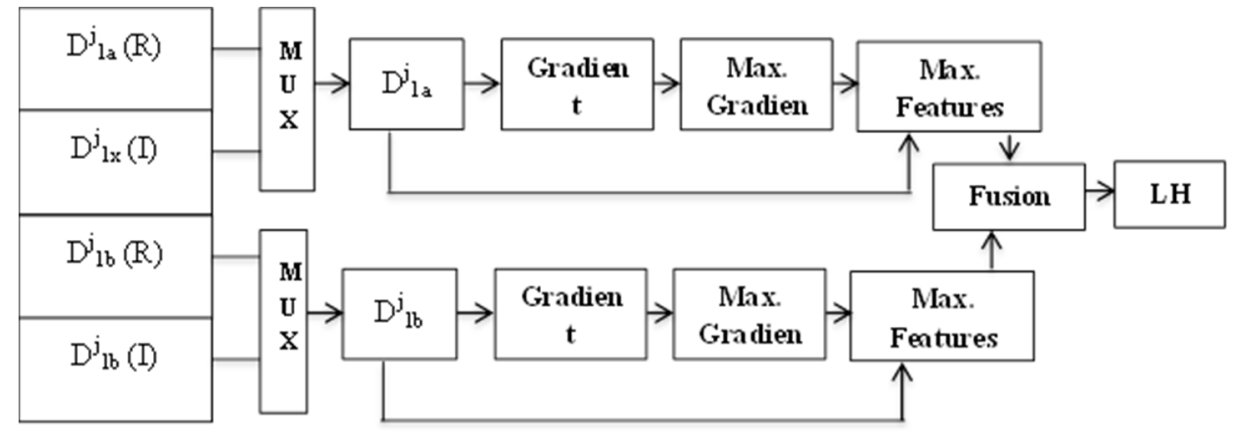

Figure 7: Companding algorithms for higher sub band (LH)

The companding algorithm generates one low pass sub bands $L L$ and three high pass sub bands $L H, H L$ and $\mathrm{HH}$ after level-1 decomposition. Similarly from the second level and third level DTCWT sub bands four DWT equivalent bands are generated to give rise to three level DWT bands. The SPIHT encoding algorithm encodes the DWT bands to bit stream achieving compression. 


\subsection{Modeling and validation}

The proposed algorithm is modeled in MATLAB and the input image is designed to contain edges in all directions. The test data input is first decomposed using DTCWT filters shown in Table 1 and Table 2. The first level decomposition gives rise to sixteen sub bands (shown in Figure 8). From the sixteen sub bands, it is found that the low pass sub bands are identical in terms of intensity. The twelve higher sub bands contain edges in different orientations. The DTCWT output pair \{High Pass 1 Real, High pass 1 Imaginary\}, contain vertical edges, the DTCWT output pair \{High pass 2 Real, High pass 2 Imaginary\} contain horizontal edges. Similarly it is observed from the other sub band outputs, that the edges in different orientations are captured in either real or imaginary band. Thus, the companding algorithm chooses one of the bands (either real or imaginary) from the pair of sub bands and is fused with the other image to obtain the DWT equivalent bands.

\subsection{Results \& Discussion}

The proposed algorithm is designed based on appropriate filters for forward DTCWT and inverse DWT computation selected from Table 1 and Table 2. DTCWT bands for three levels are computed and are companded to three levels of DWT equivalent bands. The companded bands are quantized and are encoded using SPIHT algorithm. The compressed bit stream is used to compute the compression ratio. The inverse process consists of inverse SPIHT, inverse quantization and inverse DWT.

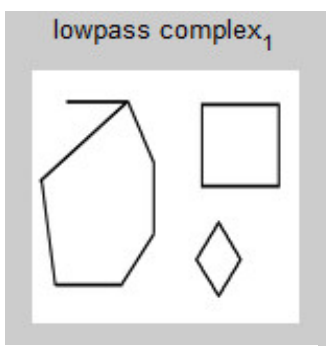

(a)

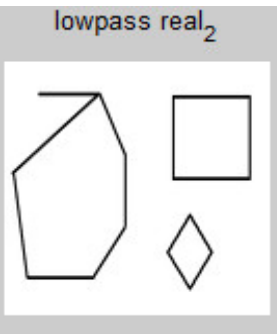

(b)

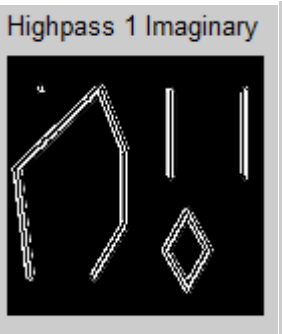

(c)

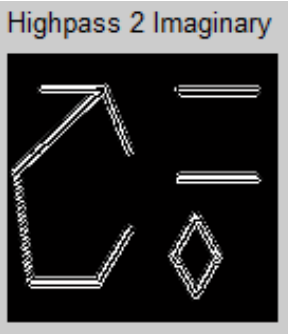

(d)

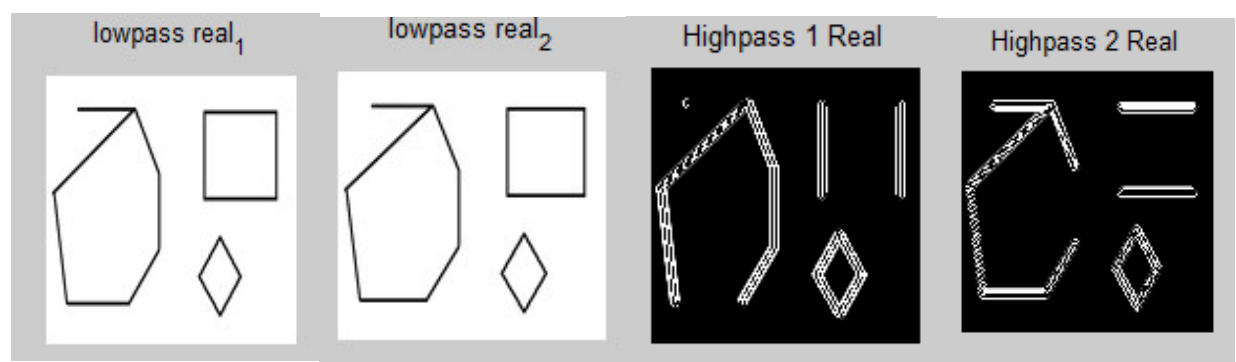

(e)

(f)

(g)

(h)

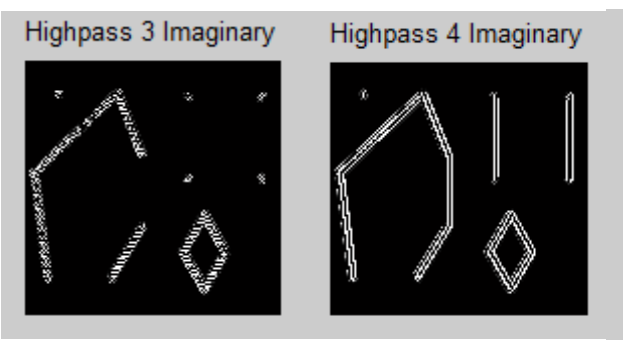

(i) (j)

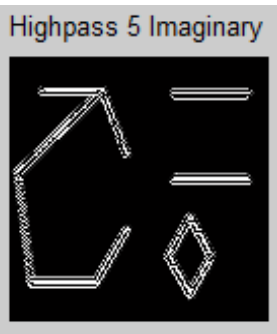

(k)

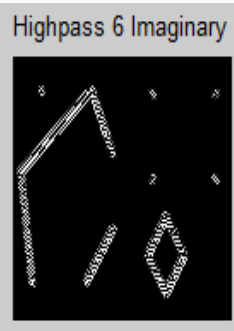

(I) 


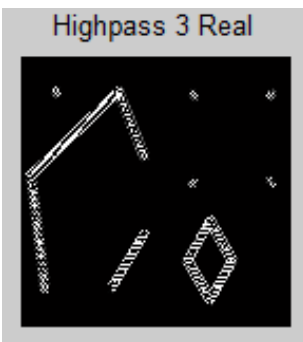

(m)

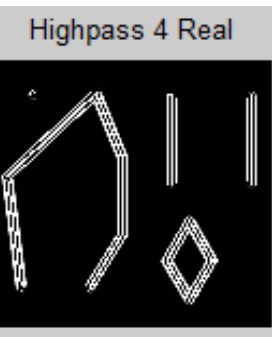

(n)

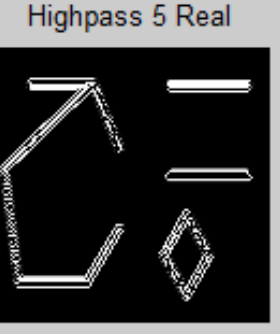

(o)
Highpass 6 Real

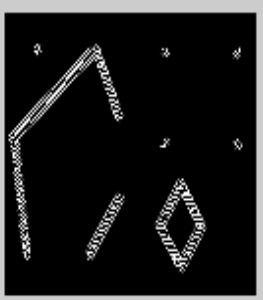

(p)

Figure 8: DTCWT sub bands for test image

Compression metrics such as MSE and PSNR and compression ratio are computed and is presented in Table 4.

Table 4 Comparison of compression results

\begin{tabular}{|c|c|c|c|c|c|c|}
\hline \multirow{2}{*}{ bpp } & \multicolumn{3}{|c|}{ Proposed Compression algorithm } & \multicolumn{3}{c|}{ DWT } \\
\cline { 2 - 7 } & MSE & PSNR & $\begin{array}{c}\text { Compression } \\
\text { Ratio DTCWT }\end{array}$ & MSE & PSNR & $\begin{array}{c}\text { Compression } \\
\text { Ratio DWT }\end{array}$ \\
\hline 4 & 0.3988 & 51.1751 & 56.5357 & 0.3963 & 52.1504 & 49.2058 \\
\hline 3 & 0.3981 & 51.1747 & 57.4841 & 0.3963 & 52.1504 & 49.2058 \\
\hline 2 & 0.3892 & 50.7761 & 63.5078 & 0.3884 & 52.2376 & 49.9035 \\
\hline 1 & 0.7219 & 49.1782 & 69.4071 & 0.7100 & 49.6183 & 56.2967 \\
\hline 0.5 & 1.411 & 47.1800 & 71.9852 & 1.2016 & 47.3331 & 62.3787 \\
\hline 0.4 & 1.734 & 45.0818 & 73.6671 & 1.6759 & 45.8884 & 65.3580 \\
\hline 0.3 & 2.2911 & 44.1838 & 74.8079 & 2.1839 & 44.7385 & 67.5323 \\
\hline 0.2 & 2.797 & 43.1857 & 75.6041 & 2.6852 & 43.8410 & 68.8852 \\
\hline 0.1 & 3.4241 & 42.1888 & 76.2663 & 3.2247 & 43.0459 & 70.0787 \\
\hline 0.075 & 3.896 & 41.9201 & 76.7721 & 3.7596 & 42.3794 & 70.9753 \\
\hline 0.05 & 4.8521 & 41.1947 & 77.1345 & 4.3247 & 41.7712 & 71.7905 \\
\hline 0.01 & 5.325 & 40.8977 & 77.4591 & 4.9308 & 41.2017 & 72.4834 \\
\hline
\end{tabular}

From the results presented in Table 4, it is observed that proposed algorithm is able to achieve higher compression at low bits per pixel (bpp). With regard to compression the proposed algorithm outperforms DWT based compression algorithm by $35 \%$. In order to evaluate the performance of image reconstruction in terms of reconstructing all features, reconstruction is carried out with DWT and DTCWT filters for the selected bands.

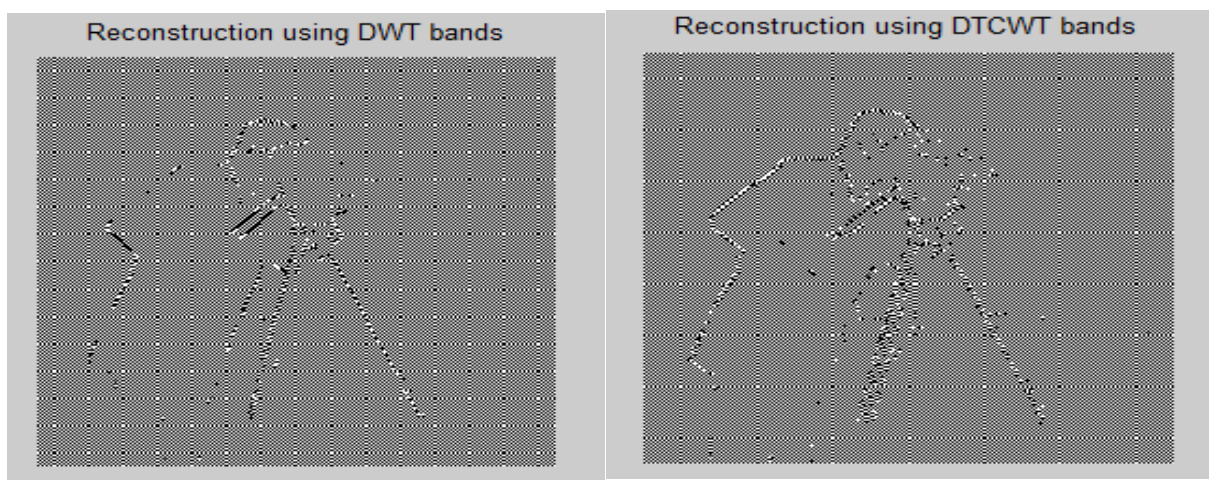

Figure 9: comparison of reconstructed output 
From the reconstructed results shown in Figure 9, DTCWT output (Figure 9(a))is able to reconstruct all features as compared with DWT results Figure $9(\mathrm{~b})$. Thus the proposed compression algorithm is able to retrieve all the required features during decomposition and increases redundancies in the DTCWT coefficients, thus enhancing the compression ratio. However, it is required to design appropriate filters that satisfy perfect reconstruction property for image reconstruction to improve PSNR.

\section{Conclusion}

Image compression is a process that is performed in three steps: transformation, quantization and encoding. Quantization introduces loss, transformation and entropy coding are lossless. Quantization of transformed bands leads to loss of significant features in the image during reconstruction. In this work, in order to minimize the effects of quantization and improve compression ratio, DTCWT is used to decompose image into multiple band. Each sub band consists of orientations in six directions in both real and imaginary parts. The companding algorithm captures all these orientations and reduces the number of bands to three high pass bands. The companded low pass and high pass bands are encoded using SPIHT algorithm thus improving compression ratio. The compressed bit stream is decoded with SPIHT decoder and the original image is reconstructed using DWT that uses DTCWT filters. As the reconstruction is based on DWT, the computation complexity in decoder is reduced by $75 \%$ as compared with DTCWT decoder. The selection of DTCWT filters for decomposition and reconstruction need to be appropriate to achieve higher PSN

\section{REFERENCES}

[1] JPEG2000 Image Compression Fundamentals, Standards and Practice: Image ...By David Taubman, Michael Marcellin

[2] Grzegorz Pastuszak; Andrzej Abramowski IEEE Transactions on Circuits and Systems for Video Technology (Volume: 26, Issue: 1, Jan. 2016 ), 210-222,

[3] G.J. Sullivan, J.-R. Ohm, W. Han, T. Wiegand, Overview of the high efficiency video coding (HEVC) standard, IEEE Trans. Circuits Syst. Video Technol., 22 (2012), pp. 1649-1668

[4] D. Taubman, "High Performance Scalable Image Compression with EBCOT ," IEEE Trans.on Image Processing, vol. 9, pp. 1158-1170, July, 2000

[5] Z. Xiong, X. Wu, S. Cheng, J. Hua, Lossy-to-Lossless compression of medical volumetric data using three-dimensional integer wavelet transforms, IEEE Trans. Med. Imaging, 22 (2003), pp. 459-470

[6] N.G. Kingsbury, "Complex wavelets for shift invariant analysis and filtering of signals," Journal of Applied and Computational Harmonic Analysis, vol. 10, no. 3, pp. 234-253, May 2001

[7] Li Hui Fang, Xu Hou Jie, Miao Guo Feng, "Images Compression Using Dual Tree Complex Wavelet Transform", Information Science and Management Engineering, International Conference of, vol. 01, no. , pp. 559-562, 2010, doi:10.1109/ISME.2010.213 
Venkateshappa, Cyril Prasanna Raj P; Design of DTCWT-DWT Image Compressor-Decompressor with Companding Algorithm. Advances in Image and Video Processing, Volume 5 No 1, February (2017); pp: 1-12

[8] Fowler James E, Joseph B. Boettcher and B'eatrice Pesquet-Popescu "Image Coding using Complex Dual Tree Wavelet Transform" 15th European Signal Processing Conference (EUSIPCO 2007),/ @2007 EURASIP

[9] Chuo-Ling Chang and Bernd Girod, "Direction Adaptive Discrete Wavelet Transform for Image Compression", IEEE Transactions on Image Processing, VOL. 16, NO. 5, MAY 2007

[10] J. Yang, J. Xu, F. Wu, Q. Dai, and Y.Wang. "Image Coding Using 2D Anisotropic Dual Tree Discrete Wavelet Transform" IEEE 2007

[11] Hasan Demirel; Gholamreza Anbarjafari, "Satellite Image Resolution Enhancement Using Complex Wavelet Transform", IEEE Geoscience and Remote Sensing Letters (Volume: 7, Issue: 1, Jan. 2010$), 123-126$

[12] Hilal Naimi , Amel Baha Houda Adamou-Mitiche, Lahcène Mitiche, Medical image denoising using dual tree complex thresholding wavelet transform and Wiener filter, Journal of King Saud University - Computer and Information Sciences Volume 27, Issue 1, January 2015, pp. 40-45

[13] N. G. KINGSBURY. A dual-tree complex wavelet transform with improved orthogonality and symmetry properties. In Proceedings of the IEEE Int. Conf. on Image Proc. (ICIP), 2000

[14] Julia Neumann Gabriele Steidl, "Dual Tree ComplexWavelet Transform in the Frequency Domain and an Application to Signal Classification"

[15] Ivan W. Selesnick, Richard G. Baraniuk, and Nick G. Kingsbury, "The Dual Tree Complex Wavelet Transform”, IEEE SIGNAL PROCESSING MAGAZINE NOVEMBER 2005, pp.123-151

[16] Ivan W. Selesnick, Richard G. Baraniuk, and Nick G. Kingsbury, "The Dual Tree Complex Wavelet Transform", IEEE SIGNAL PROCESSING MAGAZINE NOVEMBER 2005, 123-151

[17] J.M. Shapiro, "Embedded image coding using Zero trees of wavelet coefficients," IEEE Trans. on Signal Processing, vol.41, pp. 3445-3462, Dec.,1993

[18] A.Said and W.A.Perlman, "A New, Fast, and Efficient Image Codec Based on Set Partitioning in Hierarchical Trees," IEEE Trans. on Circuits and Systems for Video Technology, vol.6, pp. 243250, June, 1996

[19] D. Taubman, "High Performance Scalable Image Compression with EBCOT ," IEEE Trans.on Image Processing, vol. 9, pp. 1158-1170, July, 2000.

[20] Image Gradients Class Notes for CMSC 426, Fall 2005 David Jacobs, http://www.cs.umd.edu/ djacobs/CMSC426/ImageGradients.pdf 